\title{
A mathematical tonic
}

\section{Dr Euler's Fabulous Formula: Cures Many Mathematical IIIs \\ by Paul Nahin \\ Princeton University Press: 2006. 404 pp. $\$ 29.95, \notin 18.95$}

\section{Timothy Gowers}

One of the effects of the remarkable success of Stephen Hawking's A Brief History Of Time (Bantam, 1988) was a burgeoning of the market for popular science writing. It did not take long for mathematics to get in on the act, especially when Andrew Wiles captured the public imagination by proving Fermat's last theorem. Now scarcely a month goes by without a new popular mathematics book. Some are about famous unsolved problems, others are about notable numbers such as zero, $\pi$ or $e$, and still others, including Dr Euler's Fabulous Formula by Paul Nahin, are about important equations.

Mathematics is not easy to explain, and many authors writing popular mathematics go out of their way not to lose their readers, perhaps bearing in mind Hawking's famous dictum that each equation included in a book will halve the sales. But what if, like most readers of Nature, you already know some mathematics? Then a book that avoids mathematical symbolism is likely to be frustratingly vague. Fortunately, however, among the books that have appeared recently, there are some that might be called 'semi-popular': they are still rather informal but are aimed at a more mathematically sophisticated audience. Dr Euler's Fabulous Formula is a welcome addition to this category.

The hero of the book is Leonhard Euler (1707-83), who was one of the most prolific mathematicians ever, continuing to publish large numbers of papers even after going blind in 1766. The "fabulous formula" of the title is $e^{i \pi}=-1$, together with the more general $e^{i \theta}=\cos \theta+i \sin \theta$. These equations astonish each new generation of mathematicians that encounters them: without generous helpings of hindsight, who would ever have suspected there was such a succinct equation involving $e, i$ and $\pi$ ? Nahin is not particularly concerned with the philosophical problems associated with complex numbers, which are discussed in his earlier, less technical book An Imaginary Tale: the Story of $\sqrt{ }-1$ (Princeton University Press, 1998). Rather, he takes Euler's formula as his starting point and demonstrates the wide range of uses to which it has been put.

As an engineer, Nahin's perspective on the formula is different from that of a typical mathematician. This is mostly a good thing: not many mathematicians know much about signal processing or the importance of complex numbers to engineers, and it is fascinating to find out about these subjects. Furthermore, it is likely that a mathematician would have got hung up on details that Nahin cheerfully, and rightly, ignores. However, his background has the occasional disadvantage as well, such as when he discusses the matrix formulation of de Moivre's theorem, the fundamental result

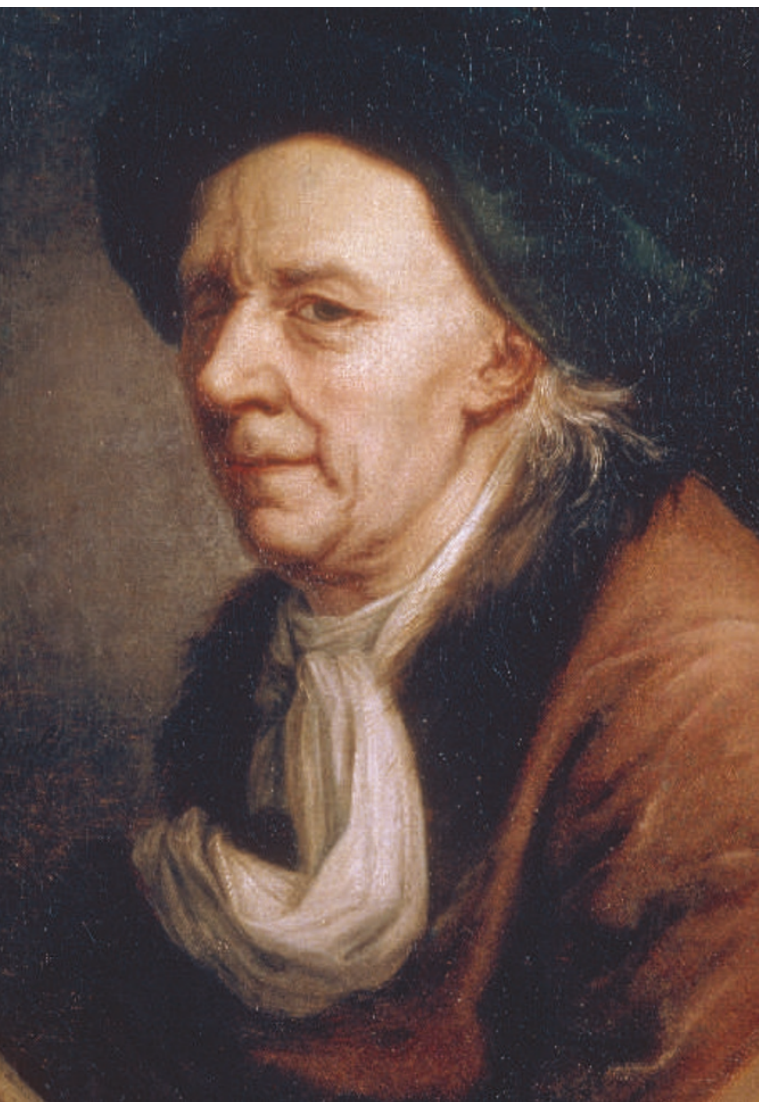

eonhard Euler linked some fundamental mathematical constants.

reader nonplussed. For instance, he discusses a problem in which a mouse runs at constant speed round a circle, and a cat, starting at the centre of the circle, runs at constant speed directly towards the mouse at all times. Does the cat catch the mouse? Yes, if, and only if, it runs faster than the mouse. In the middle of the discussion Nahin suddenly abandons the mathematics and puts a discrete approximation into his computer to generate some diagrams. His justification is that an analytic formula for the cat's position is hard to obtain, but actually the problem can be neatly solved without such a formula (although it is good to have his diagrams as well).

A question Nahin does not engage with is whether complex numbers are indispensable to his arguments. Such is his enthusiasm for complex numbers that he writes as though they always are. But there is a big difference between an argument that uses both addition and multiplication of complex numbers and one that uses just addition (in which case the complex numbers are just a convenient notation for the real plane). For example, he uses them in his analysis of the cat-and-mouse problem above, but he needn't have done. Elsewhere he explicitly says that he has used complex numbers in a fundamental way, when he hasn't. For instance, to demonstrate that a certain quadratic equation has no real solution, he points out that the familiar formula for the roots shows that they are both complex. This is not a true use of complex numbers, because to derive the formula for the roots one must complete the square, and then the true reason for the lack of real roots emerges: the square of a real number is never negative.

On the whole these defects are not serious, however. Indeed, mathematicians may well find them thoughtprovoking and others will not notice

that $(\cos \theta+i \sin \theta)^{n}=\cos (n \theta)+i \sin (n \theta)$. This is a one-line consequence of the addition formulae for sines and cosines, but his proof takes up an incredible six pages and uses, quite unnecessarily, the Cayley-Hamilton theorem, which he proves in two dimensions by brute force. Even then the proof is not complete: at one point he says he will "refer you to any good book on linear algebra for a formal proof”. The less experienced reader will emerge from these pages thinking that de Moivre's theorem is a dauntingly difficult result, which is a pity, as it isn't.

Sometimes Nahin reveals his engineer's instincts by dispensing with formal proof and using numerical simulations instead. In places this works well - if a result is plausible a proof isn't always needed in a book like this - but sometimes it can leave a mathematical them. On the positive side, Nahin includes gems from all over mathematics, ranging from engineering applications to beautiful pure-mathematical identities. Most of his topics lie just beyond the periphery of a typical mathematics course: they are facts, such as the irrationality of $\pi$, that you may have heard of but never had explained in detail. It would be good to have more books like this.

Timothy Gowers is at the Centre for Mathematical Sciences, University of Cambridge, Wilberforce Road, Cambridge CB3 OWB, UK.

\section{Correction}

In the review of the book Soils and Societies: Perspectives from Environmental Histories (Nature $442,985 ; 2006)$, the name of one of the editors was misspelled. The editors were J. R. McNeill and Verena Winiwarter. 